\title{
Monitoring the Best Operating Point of Centrifugal Pumps Using Blade Passing Vibration Signals
}

\author{
A. Eaton, W.H. Ahmed, M. Hassan \\ University of Guelph \\ 50 Stone Rd E, Guelph, Canada \\ eatona@uoguelph.ca; ahmedw@uoguelph.ca; mahassan@uoguelph.ca
}

\begin{abstract}
Degradation of centrifugal pumps can occur over time and can often lead the pump to operate away from best efficiency point. Therefore, it is necessary to utilize monitoring techniques to ensure the proper operation. In this work, the effect of operating the pump away from the best efficiency point on the vibration characteristics is investigated. An industrial centrifugal pump rated at $7.5 \mathrm{~kW}$ is installed in a flow testing loop to determine the pump performance and the vibration response at the full operational range the pump. The vibration response was measured using accelerometers placed on the suction and discharge flanges of the pump casing. The amplitude of the blade passing frequency represented by FFT spectrum is determined for various flow rates. The minimum amplitude of the blade passing frequency was found to occur at a flow rate of $17 \mathrm{~L} / \mathrm{s}$. Meanwhile, the entire performance of the pump was evaluated and found that the best efficiency of $72.5 \%$ occurs at $17 \mathrm{~L} / \mathrm{s}$. Furthermore, the pump efficiency was found to decrease as the amplitude of the blade passing frequency increases. The results show that the amplitude of the blade passing frequency can be used to help monitor the pumps best operating point.
\end{abstract}

Keywords: Centrifugal pump, Flow rate, Vibration, Blade passing frequency.

\section{Introduction}

Centrifugal pumps are used in a variety of engineering applications such as power production, heating and cooling, and water distribution. Although centrifugal pumps are considered a highly reliable product, they are susceptible to many forms of degradation and damage due to harsh environment, poor design, incorrect operating conditions and improper installation. Therefore, it is necessary to employ condition monitoring techniques in order to predict possible failures. The most common form of condition monitoring for rotating machinery is vibration analysis [1]. Examining the vibration spectrum in the time and frequency domain is frequently used to determine operating faults. Furthermore, spectral analysis can provide more detailed information regarding the mechanical condition of the pump [2].

The vibration characteristics are affected not only by degradation but also with the changes in the flow characteristics. It is therefore necessary to identify and differentiate how different operating conditions manifest as vibration and affect the performance of the pump. Several different factors can go into vibration monitoring of rotating machinery. For centrifugal pumps, two main categories of vibration sources exist: Mechanical and Hydraulic [3], [4]. Mechanical factors include: imbalance, bent, misaligned shaft or bearing misalignment. While the hydraulic factors are comprised of: hydraulic imbalance, vane passing forces, internal recirculation, suction and discharge recirculation, rotating stalls and cavitation [3], [4].

Characteristics of the vibration spectrum for the various factors have been outlined in [3], [4]. Frequencies of the vibration spectrum that correspond to the rotational frequency and its harmonics are known to account for the mechanical effects such as an imbalance. Hydraulic effects are most commonly found to manifest as changes in the pressure fluctuations which can be illustrated by the blade passing frequency and its harmonics in the FFT spectrum. The effects of flow rate on a double suction centrifugal pump are investigated by [4]. It was found that the vibration decreases when approaching the best efficiency point. However, the measurements were taken from the shaft bearing. Pressure fluctuations and operating point is investigated by [5]. Also, dynamic pressure signals are used to measure the pressure fluctuations at various locations of the pump volute. It was found that the dominating frequency in the spectrum corresponded to the blade passing frequency. Furthermore, the minimum pressure fluctuations occurred at roughly $80 \%$ of the best efficiency flow rate for all rotational speeds investigated. The vibration characteristics of centrifugal pumps with emphasis on the blade passing frequency is 
discussed in detail by [6]. The pressure pulsations are attributed to the impeller blade wake as it passes the stationary volute leading edge. Furthermore, the energy produced by the pressure pulsations are dissipated through structural vibration. These pressure pulsations are known to increase at partial flow rates and at flows above best efficiency point but at a lesser extent. Several problems can arise due to pressure pulsations at the blade passing frequency that include: repeated dynamic loading which can lead to fatigue over time, breakage of the impeller shroud and noise [6].

In this paper, the effects of pressure pulsations on the vibration characteristics of an industrial centrifugal pump are investigated by varying the pump flow rate. Furthermore, the results from this work can be used as a baseline for comparison when various forms of damages are encountered in the pump components.

\section{Experimental Setup and Methodology}

The centrifugal pumps are installed in a state-of-the-art testing rig that can accommodate two pumps, variable speed drive, and pipe network. The pumps being investigated are vertical inline, split coupled centrifugal pumps provided by Xylem Inc. Each pump is equipped with a $23.5 \mathrm{~cm}$ diameter stainless steel impeller. Two pumps are installed in a parallel arrangement in the experimental setup. At the nominal speed of $1750 \mathrm{RPM}$, each pump can produce $17 \mathrm{~L} / \mathrm{s}$ and $206.8 \mathrm{kPa}$ when operating at the best efficiency point. Furthermore, the maximum flow rate is $24.6 \mathrm{~L} / \mathrm{s}$ and maximum pressure is 262 $\mathrm{kPa}$ when operating at the nominal speed. Each pump is connected to a variable speed drive that can change the speed of the pump to a minimum value of 900 RPM.

Due to the wide range of operating points for centrifugal pumps, a piping system was designed to test pumps at many operating points. The primary loop is designed to accommodate the full operational range of the pump as the majority of the pipe is 3-inch 316 stainless steel to minimize the pressure losses in the system. To provide a reservoir for the flow loop a 1000 L 304 stainless steel tank was custom fabricated and installed at the end of the pipe line. Some additional loop components include an air and dirt separator to remove undesired air and particulates from the system, an expansion tank to provide pressure relief due to thermal expansion and a compressor to provide enough positive pressure on the suction side of the pump. Water is used as the working fluid for this work and a flow diagram that describes the system can be seen in Figure 1a. The pump flow rate is measured by an $\mathrm{OMEGA}^{\odot}$ ultrasonic flow meter capable of measuring flow up to $25.3 \mathrm{~L} / \mathrm{s}$. Suction and discharge pressure of the pumps are measured by high accuracy OMEGA ${ }^{\odot}$ pressure transducers. The suction measurement range is from $\pm 103.4 \mathrm{kPa}$ while the discharge pressure transducer measures the range of 0-344.7 $\mathrm{kPa}$. In order to measure the actual input power to the pump, the shaft torque and rotational speed is measured. Dynamic torque acting on the pump shaft is also measured using a strain gauge that is connected to a signal transmitter. The transmitter is mounted onto the motor/pump shaft coupling and transmits the torque signal while the pump is operating. Rotational speed is measured using an infrared tachometer which also acts as the signal receiver for the torque measurement. Two Dytran ${ }^{\odot}$ accelerometers are also installed to measure the vibration of the pumps. An accelerometer is installed on the suction and discharge pipes in the plane of impeller rotation, each with a sensitivity of $10 \mathrm{mV} / \mathrm{g}$. 


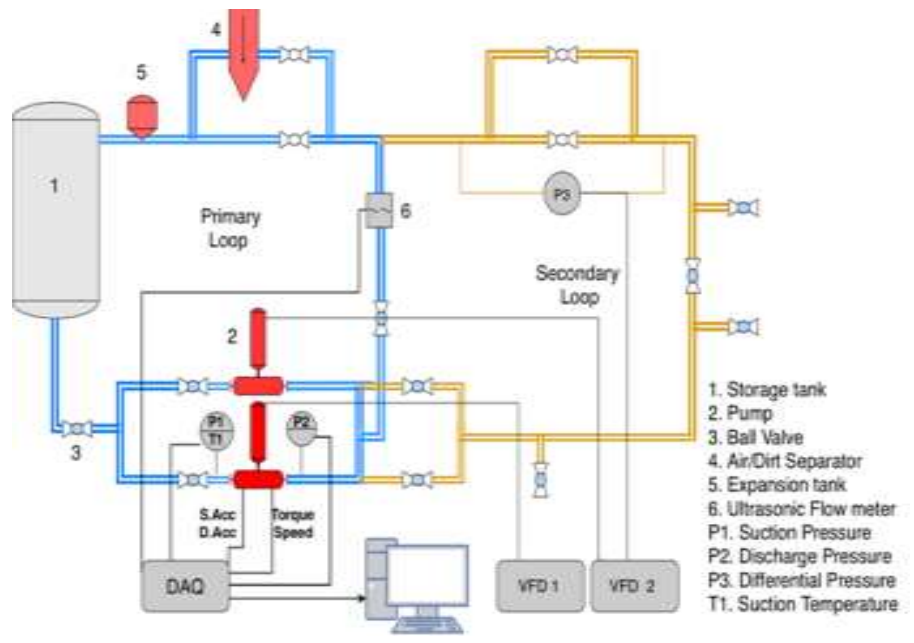

a) Schematic flow diagram

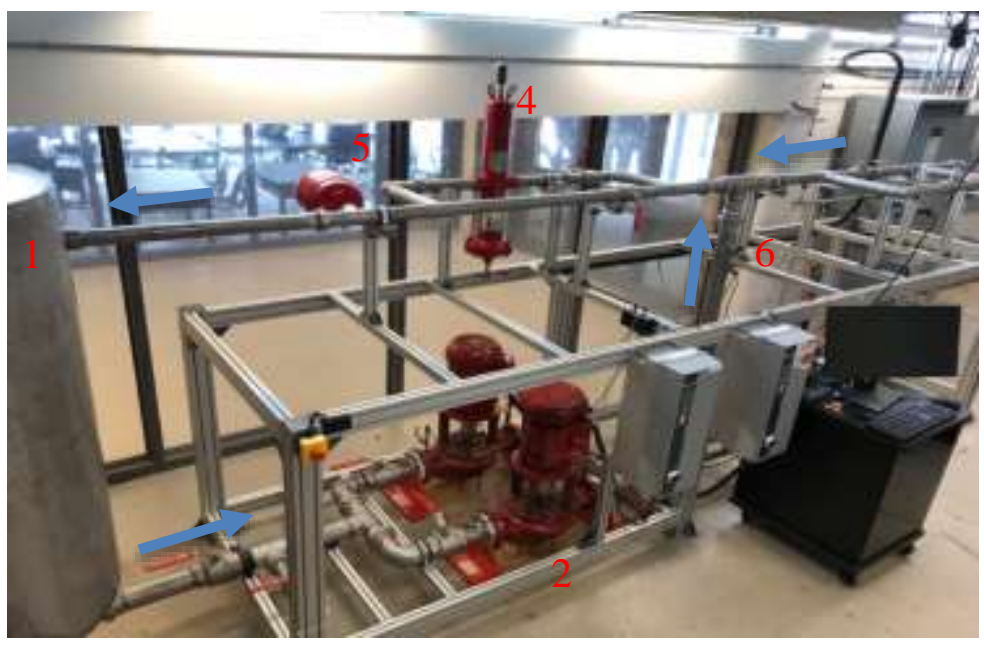

b) Actual Image of the pump setup

Fig. 1: Experimental Setup.

It should be noted that the measurement uncertainty is $\pm 1 \%$ of the reading for the pressure transducers and the flow meter while the uncertainty of the torque measurement is $\pm 0.5 \%$. This leads to an uncertainty of $\pm 1.4 \%$ and $\pm \pm 1.8 \%$ for the pump head and the efficiency respectively. Furthermore, the uncertainty of the accelerometer sensitivity is $\pm 10 \%$. Experiments were performed for the full operational range of the pump. Flow rate was varied from $0-22.7 \mathrm{~L} / \mathrm{s}$ at an interval of $1.9 \mathrm{~L} / \mathrm{s}$ to construct an accurate pump curve. For each operating point 5 samples were taken for 60 seconds at a sampling rate of $3 \mathrm{kHz}$. The experimental data was then processed in MATLAB.

\section{Results and Discussion}

The performance of the pump was measured for the entire range of pump operation. Flow rate was varied and pressure, shaft power and acceleration were recorded. The differential pressure and the pump efficiency are determined and plotted in Figure 2. It can be seen that the best efficiency point of the pump is at $17 \mathrm{~L} / \mathrm{s}$ and reaches a value of $72.5 \%$. This is slightly different than the manufacturing specifications, but the difference can be attributed to manufacturing tolerances. 


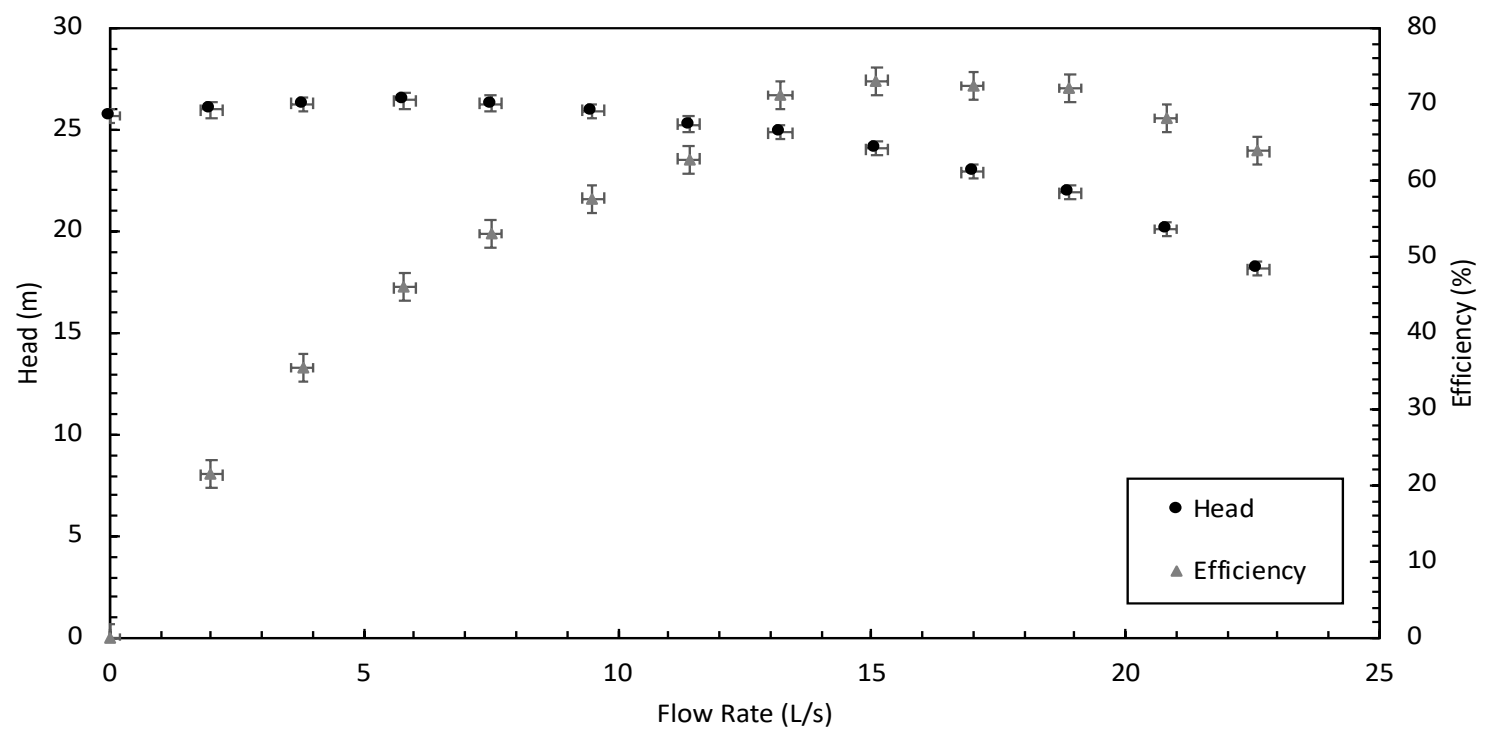

Fig. 2: Measured pump performance curve.

The vibration characteristic being considered is the amplitude of the peak that corresponds to the blade passing frequency in the FFT spectrum. A sample of the acceleration signal that corresponds to the pump operating at $17 \mathrm{~L} / \mathrm{s}$ is shown in Figure 3 and the corresponding FFT spectrum in Figure 4. It can be seen that the most dominant peak is at $29 \mathrm{~Hz}$ which corresponds to the nominal operating speed, $\mathrm{N}$. Its harmonics $(2 \mathrm{~N}, 3 \mathrm{~N}, 4 \mathrm{~N}, 5 \mathrm{~N})$ can also be seen but at a much smaller amplitude. Peaks at these frequencies can be attributed to mechanical factors such as an imbalance.

Since the impeller used for this work is designed with 7 blades, the blade passing frequency can be found at $7 \mathrm{~N}$, which corresponds to $203 \mathrm{~Hz}$. The amplitude blade passing frequency is used the compare the vibration measurements to the performance in order to establish a correlation between pump efficiency and the vibration response. Since blade passing frequency is known to be affected by suction and discharge pressure pulsation it can be affected by the change in flow rate [6]. Flow rates that range from $9.5 \mathrm{~L} / \mathrm{s}$ to $22.6 \mathrm{~L} / \mathrm{s}$ are considered for the vibration analysis. The variations in the peaks at blade passing frequency can be found in Figure 5. It can be seen that there is a decrease in the blade passing frequency peaks from $9.5 \mathrm{~L} / \mathrm{s}$ to $17.1 \mathrm{~L} / \mathrm{s}$ when looking at the suction acceleration data. For larger flow rates the blade passing frequency amplitude starts to increase.

Comparing the vibration and performance results, it can be seen that the maximum pump efficiency and the minimum vibration at blade passing frequency occur at $17 \mathrm{~L} / \mathrm{s}$. Furthermore, as the efficiency drops, the blade passing frequency increases. This indicates that at the best efficiency point the amplitude of the blade passing frequency will be at its minimum for the suction acceleration. It is important to note that the vibration increases at a higher rate when the flow is decreasing 


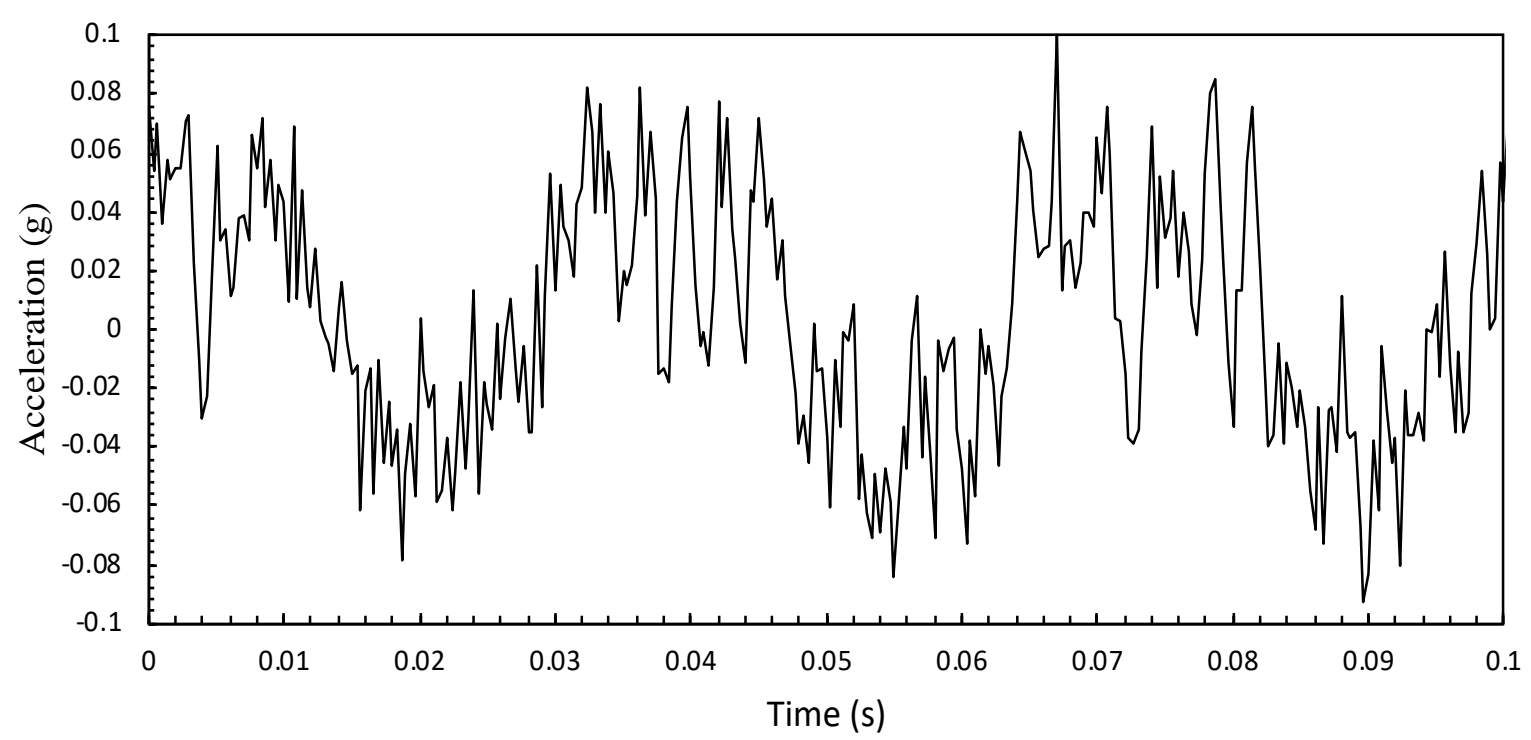

Fig. 3: Sample of suction acceleration signal at $17 \mathrm{~L} / \mathrm{s}$.

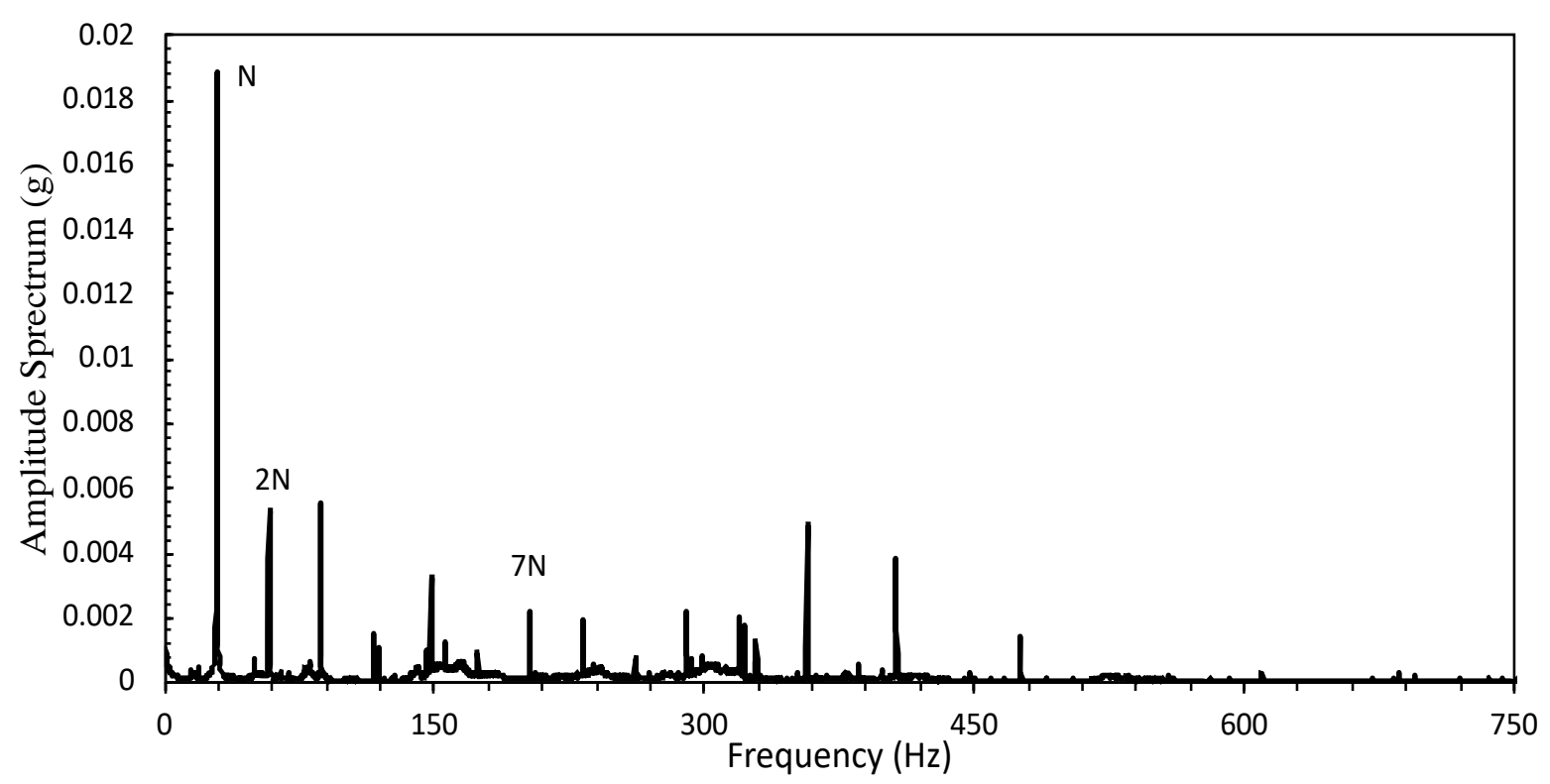

Fig. 4: Suction acceleration FFT spectrum at $17 \mathrm{~L} / \mathrm{s}$.

from the best efficiency point, when compared to increasing the flow rate. These trends correlate well with the pressure pulsation characteristics described by [6]. Therefore, monitoring of the vibration amplitude at blade passing frequency can indicate any changes in the pressure pulsations within the pump and consequently deviations from the best efficiency point. 


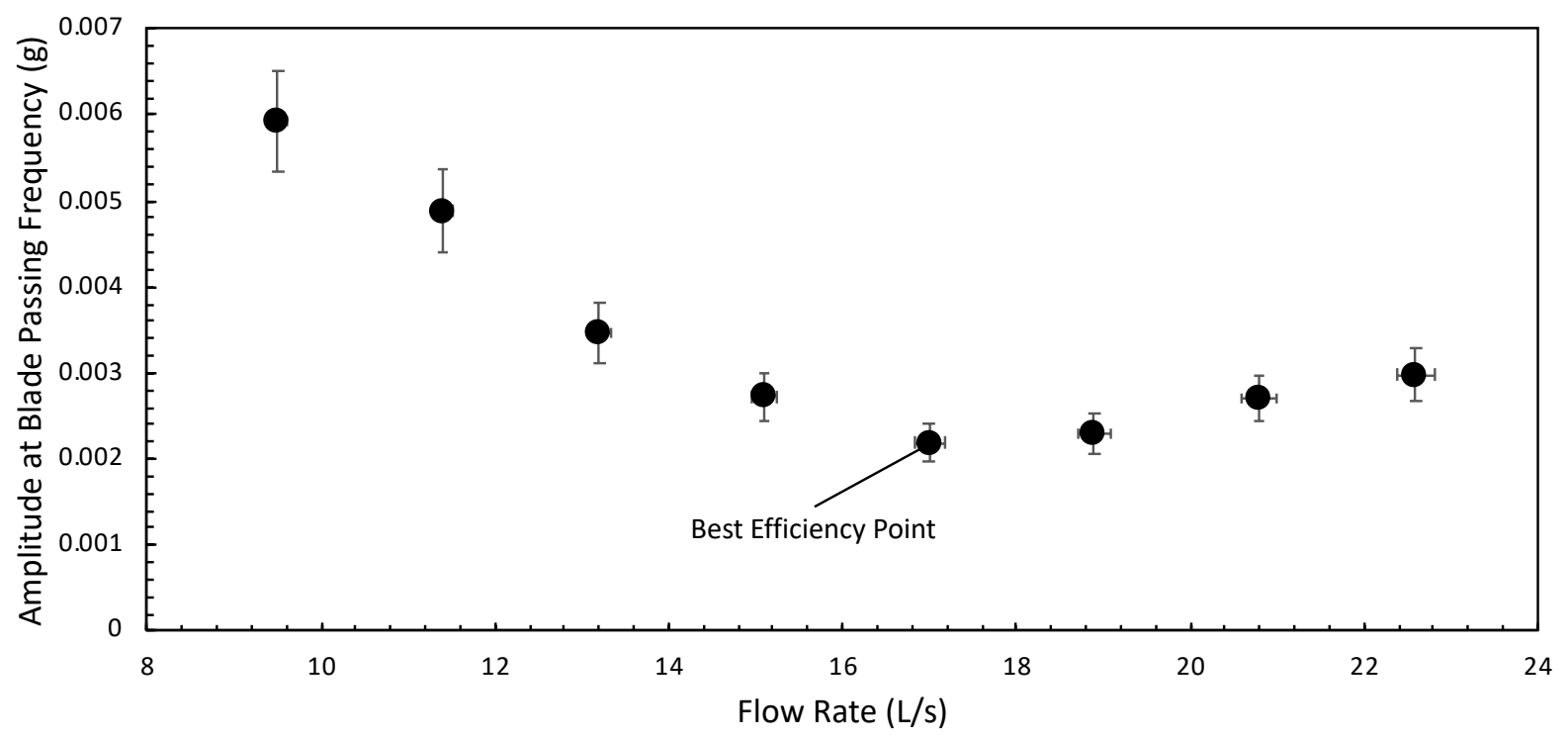

Fig. 5: Amplitude of blade passing frequency at different flow rates.

\section{Conclusion}

The results show that the amplitude of the blade passing frequency can be used to identify whether the pump is operating at the best efficiency point or not. Furthermore, the changes in pressure pulsations due to changes to the flow rates can be detected using vibration analysis. The results obtained from this work will act as a baseline trend to be used for when damage is introduced to the impeller. Any major changes in the frequency spectrum and the amplitude at the blade passing frequency from this baseline trend can be used to identify possible forms of damage in pumps.

\section{Acknowledgement}

The authors would like to acknowledge the support of Natural Sciences and Engineering Research Council of Canada (NSERC). The support of Xylem AWS is greatly appreciated.

\section{References}

[1] R. S. Beebe, Predictive Maintenance of Pumps Using Condition Monitoring. Elsevier Science and Technology Books, 2004.

[2] D. A. Casada, "Detection of Pump Degradation," in 22nd Water Reactor Safety Information Meeting, 1994.

[3] F. Stefan and F. Arno, "Dynamic Loading on Pumps - Causes for Vibration," in Proceedings of the Tenth International Pump User Symposium, 1993, pp. 171-184.

[4] M. R. Hodkiewicz and M. P. Norton, "The effect of change in flow rate on the vibration of double-suction centrifugal pumps," in Proc. Inst. Mech. Eng. Part E J. Process Mech. Eng., 2002, vol. 216, no. 1, pp. 47-58.

[5] J. L. Parrondo-Gayo, J. González-Pérez, and J. Fernández-Francos, "The Effect of the Operating Point on the Pressure Fluctuations at the Blade Passage Frequency in the Volute of a Centrifugal Pump," J. Fluids Eng., vol. 124, no. 3, p. 784, 2002.

[6] F. L. Robinett, J. F. Gulich, and T. Kaiser, "Vane Pass Vibration - Source Assessment and Correction -A Practical Guide For Centrifugal Pumps," in Proceedings of the 16th International Pump Users Symposium, 1999, pp. 121-138. 